second investigation entirely confirms the accuracy of the first as regards the periodic inequalities, but that the secular variations of the excentricity and longitude of the perihelion are slightly changed. The effect of these changes is to bring the theory into very satisfactory accordance with the observations of Bradley, but the discrepancies above mentioned in the time of Maskelyne and in the modern observations still remain unaffected. The character of the discrepancies shown by the modern observations makes it very improbable that they can be due to any errors in the theory.

In fact, the error appears 10 change almost suddenly from a positive one of $4^{\prime \prime} \cdot 4$ in 1839 to a negative one of $5^{\prime \prime} \circ$ in 1844 , a variation of nearly $9 " 5$ in five years. Now no terms or group of terms due to the action of the planets could thus suddenly disturb the motion in five years, at a given epoch, and then leave the motion unaffected during the following twenty-five years. $M$. Leverrier is therefore inclined to think that the discrepancies arise from errors in the observations, notwithstanding that the Greenwich and Paris observations are mutually confirmatory of each other.

He suggests that it is possible that the varying aspects presented at different times by the ring may affect the accuracy of the observations of the planet, and may cause changes in the personal equations of the observers, which, from being rather large in the case of the ancient observations, have gone on diminishing as the system of observation has become more perfect.

One unlooked-for result follows from M. Leverrier's comparison of his theory of Saturn with the observations. Considering that the influence of Jupiter on the longitude of Saturn may amount to $3800^{\prime \prime}$, it might have been expected that from observations of the planet extending over I 20 years the mass of Jupiter could have been determined with great precision. M. Leverrier has found, however, that this is not the case.

The equations of condition furnished by the comparison of the heliocentric longitudes of Saturn as deduced from theory and observation contain five unknown quantities, viz,, the corrections of the assumed values of four elements and the correction of the assumed mass of Jupiter. On solving the equations with respect to the first four unknown quantities, the corrections to be applied to the elements are found to be greatly influenced by the intermediate correction of the mass of Jupiter, and after they have been substituted in the equations of condition, the coefficients of the correction of the mass of Jupiter in great part destroy each other, nowhere amounting in the resulting equations to one-tenth part of their values in the primitive equations. Hence these equations are insufficient to determine the mass of Jupiter with any precision. Consequently, in the formation of the Tables of Saturn, M. Leverrier has employed the value of the mass of Jupiter determined by the Astronomer-Royal from his observations of the $4^{\text {th }}$ satellite.

The result which has just been noticed will appear to be less paradoxical if we consider that by far the larger part of the disturbances which Jupiter produces in the motion of Saturn is represented by the inequalities of long period which affect the mean longitude and the elements of the orbit. Now in the course of 120 years these inequalities have run through only a small part of their whole period, and therefore, during this interval, the greater part of their effects may be represented by applying changes to the several mean elements equal to the mean value of the corresponding long inequalities during the interval. It is only from the residual disturbances, which are comparatively small in amount, that any data can be obtained for the correction of the mass of Jupiter.

In the course of a few centuries, when these long inequalities, as well as the secular variations of the elements of Saturn, shall have had time to develop themselves, it will be possible to determine the mass of Jupiter from them with all desirable precision.

\section{THE GLANDULAR ORIGIN OF CONTAGIOUS DISEASES 1}

TEN years ago, on the occasion of a Congress held in this town to discuss the question of the disposal of sewage, I had the honour, at the request of the committee of management, to deliver a lecture on the subject of the poisons of the spreading or communicable diseases. An abstract of the lecture was afterwards printed by the Congress, and for a time it gained a wide circulation.

The lecture of which $I$ speak was based on a series of experimental researches which for some years previously I had been carrying out on the question of the mode of production and communication of those diseases which were anciently called plagues or pestilences, but which are now called communicable or spreading diseases.

I do not think that at a health congress like the present I can do better than recall attention to this same subject. The suppression of plagues is one of the grondest and supremest efforts of the sanitary reformer. The suppression can never be accomplished until all educated people understand the advances of modern science as to the cause and mode of origin and mode of propagation of these diseases. Whatever, therefore, tends to strike out light of knowledge on these subjects tends to elacidate, and though the spark lighted may go out again it may help to show the way.

I shall in this present effort first go back to the point where I stood when here ten years ago. I shall then briefly survey the cour'se of thought that has sprung up between that time and the present. Next I shall state the position of my own views as influenced by the work of the past ten years. Lastly, I shall touch for a moment or two on the practical applications of theory to the development of practice.

\section{Outline of the Glandular Theory.}

From my researches previous to the year 1867, and which formed the subject-matter of my previous lecture here, I had discovered that the fluids secreted during various stages of disease in some forms of communicable disease could be made to propagate disease. A portion of secreted fluid taken from a patient of $\mathrm{Mr}$. Spencer Wells, a patient who was suffering from surgical fever following upon the operation of ovariotomy, had been made to produce a definite form of fever in an inferior animal by being simply brought into contact with the peritoneal surface of the animal. The secretion from the peritoneum of the affected animal was shown by further experiments to have the power of inducing the same order of phenomena of disease in other similar animals, and through four generations of animals the phenomena were repeated. These were the first experiments in which this class of phenomena of disease by direct propagation and repropagation were produced synthetically. They have since been performed and modified in many ways, and the origination of them has been assigned to different experimentalists, but I am entitled to say they were the first of the kind; they were carried out in the years I $86_{4}-6_{5}$, and they were communicated to the Association of Medical Officers of Health in the year 1865 .

During the same course of research I made an attempt to separate the poisonous matter from the poisonous secretion, and in one attempt of the sort I believed myself to have been successful. Certainly I separated a substance which was exceedingly poisonous in its action, and which, after the manner of an alkaloid, combined with

I A Theory as to the Natural or Glandular Origin of the Contagious Diseases, Address by the prosident, Benjamin W. Richardson, M.D. F.R.S., at the Sanitary Congress, Leamington, October 3 . 
acids producing salts which were not only themselves poisonous but which reproduced poisonous secretion. In the lecture delivered in this place in 1867 specimens of these salts and of the substance from which they were derived were placed on the table.

To the poisonous substance, that is to say, to the base of the poisonous matter of the communicable diseases, I gave originally the name of septine, and I classified all diseases that are induced by such substance, septinous diseases. Before this period the diseases had been named zymotic, under the idea that they were connected with a process of zymosis or fermentation. They are still commonly known by that name. Since the name I suggested was given to them, they have been called septic diseases, and the term septicæmia has been brought into use in relation to them. I am of opinion, with all respect, that the word septine, as applied to the basic poisonous matter, and the term septinous, as applied to the phenomena, are the two simplest and best terms we can employ.

As the inquiries which led up to the experiments with septine progressed, I was led to form a view as to the nature of the poisonous base and as to its mode of origin. As I have already said it seemed to me to be an alkaloid, or chemical substance resembling in physical properties morphine, strychnine, and other bodies of that class, but derived, not like them from vegetable, but from animal organic matter. The difficulty in proving this lay in finding a reason for the various effects of the septinous material. If it were a common base like that which I suspected I had found, why should it not always produce the same form of septinous disease? Why should it, on the other hand, produce, as we know it does, many kinds of disease, each having a certain general likeness to the others, but each at the same time different in many important details, as different, for example, as small-pox is from scarlet fever, or measles from hydrophobia?

The difficulty in this way suggested led me to reflect on the connection which might exist between the bases of the different secretions of the animal body and the matter of septine. Each secretion yields some organic product ; the gastric secretion pepsine, the salivary secretion ptyaline, and so on, and each secretion plays a different part in function although the organic bases of them all may present a general similitude of construction.

Thereupon I was led to the conception that the secretions of the animal body are in fact the sources of the septinous diseases, and that the various septinous diseases are, in fact, all of glandular origin; that in every case of disease the poison producing it is nothing more and nothing less than a modified form of one or other secretion.

In the lecture of I 867 delivered here a sketch was supplied of the number of diseases which affect the human tamily. They were stated to be about two hundred and fourtcen in number, that is to say, when we classify the symptoms together so as to make them into great groups to which we can give specific names, we may reckon up two hundred and fourteen such groups of diseases. Amongst these groups I described one group as depending for its cause on the action of organic poisons.

The diseases produced by the organic poisons were classed as follows :-

Small Pox.

Measles.

Scarlet Fever.

Diphtheria.

Typhus Fever.

Typhoid Fever.

Erysipelas.

ILospital Fever.

Puerperal Fever for the fever

which occurs to women in child-bed).

Cholera.

Yellow Fever.

Ague.

Glanders.

Boil and Carbuncle. Infectious Ophthalmia.

On the nature of the organic poisons which produce the diseases I urged the following points :- (a) That in every case the poisons are in themselves specific. Each poison has a specific property, always bringing out the same disease through countless ages. From the time when man was first attacked by them, on to the present time, I have no doubt that each of the communicable diseases has been developed from, and has depented upon one specific poison.

(b) That the organic poisons are inodorous, have no smell whatever, and that no communicable disease ever depends upon the mere gases of decomposition of organic matter.

(c) That as regards the organic poisons themselves and their physical properties the great type of them all is represented by the poison of any venomous snake. If we puncture the poison bag, there exudes from it a fluid substance that contains the poison. If we gently dry that down, it becomes a darkish, somewhat powdery, halfglistening mass. It is the type of all the poisons which produce disease.

(d) That the special poisons are separable, and that I had separated one of them, namely, the poison of hospital fever. This is a secretion formed in the wound of a person suffering from surgical injury, and as it could be obtained in large quantities, it had been specially selected for the purpose of experiment. The poison, when obtained in large quantities, could be evaporated to the form of an extract or syrup, and produced, when dried, a substance resembling closely the snake poison. It admitted of being pulverised, and when introduced into the wound of a healthy animal, it produced symptoms similar to those of the patient from which it was taken.

A specimen of the poison of hospital fever, so prepared was shown. It was extracted from the fluid of the peritoneal cavity of a lady who had been operated upon for ovarian disease by Mr. Spencer Wells.

(e) That the poison thus obtained may be introduced into the body in various ways; that communicated to an animal, it will give to the body of that animal the same poisonous property as was possessed by the poisonous substance first introduced; the poison, that is to say, could be passed on, and made to affect another animal, and so through a series of subjects.

$(f)$ That in the course of some diseases, these poisons are separated by nature in an almost pure state. This is singularly the case with regard to the poison of small-pox. The poison of small-pox may escape from the surface of the body, in an early stage, as a very fine vapour, and in that way communicate disease. It may be communicated in a fluid form, as we know when we use it by inoculation. In a dry state, as in the scale of a small-pox patient, it is innocuous till it comes into contact with the water or with the fluids of the body; then it becomes poisonous.

(g) That the poisons will probably dry solid. In the solid state they are inert, but they are capable of reabsorbing water apparently after any lapse of time, and of regaining their activity.

(h) That they admit of being charged with water almost to any degree; but that as we progress in charging them with water, and diluting them with water, they entirely lose their active power. This accounts for a fact which was observed by the famous Dr. Fordyce in the last century. At that time inoculation for small-pox was the rule; and Dr. Fordyce thought, "if he diluted the poison he would produce a milder form of disease." In fact, he was aiming in this way to produce what Jenner afterwards did produce by vaccination, namely, a modified smallpox. He took the poison of small-pox, mixed it with water, and refined it to a considerable extent, and he inoculated patients with the diluted solution. He then found out the fact-that, up to a given point, dilution made no difference, the poison always producing the disease; but beyond that certain point of dilution there was no disease at all produced by the solution-not even a milder disease. This was in accordance with my experiments, 
from which I found that the organic poisons retain their activity up to a given point of solution, and beyond that the water renders them inert. Through their extreme capacity for becoming watery, they lose their activity allogether.

(i) That the poisons are transferable also by the vapour of water, and in this way may escape from the living body. So long as a person is affected with these poisons, and is giving off vapour at a certain temperature, he is poisonous. The poison is distributed by the vapour, and the vapour is diffused in what I might almost call invisible spray. The poisons are mechanically carried with the vapour, and the vapour from the affected person may be absorbed by the healthy person. But as soon as the body is dead, the vaporisation having ceased, or a reverse process having been set up,--that is to say, there being a condensation of vapouras there sometimes is on the dead body, - the poisons are no longer infectious in the ordinary sense of the word.

$(j)$ That the poisons are harmless in their dry state, but commence to resume their activity in water. In order to ensure their continuous action, they need certain temperatures-certain degrees of heat; that in this respect one poison often differs materialiy from another; and that this marks out on the surface of the earth a specific range for some poisons. For instance, the poison of typhus fever is probably volatile, and condenses with difficulty, with the result that it only lives at a given low temperature, and that at a certain degree north of the equatorial line, the disease ceases. There are other poisons which require a greater degree of heat for their distribution, of which the poison of yellow fever is an example. If yellow fever be brought from a hot country to one of our northern ports, it wili not live. It may linger for a few days, but as a rule, it will not extend.

(k) That the poisons are all capable of being destroyed by various means. They are all destroyed, as already said, by extreme dilution. They are all destroyed also by heat and by oxidising agents. The mere exposure of them to moist oxygen destroys them rapidly. The exposure of them to ozonised, or electric oxygen, destroys them even more rapidly than ordinary moist oxygen. Exposure of them to chlorine is instantaneous destruction to them. Exposure to iodine is nearly as effective, and if the iodine can be diffused equally, it is as destructive as chlorine. Exposure to bromine leads to the same result. Exposure to nitrous acid has the same kind of effect, but not in so marked a degree. Exposure to sulphurous acid likewise produces destruction.

(l) That snake poison is destroyed by sunlight, and that the destruction does not depend upon the temperature That bright sunlight is probably one of the means for destroying the organic poisons.

$(m)$ That almost all the organic poisons are preservable by cold, and that, in fact, there is no limit to the preservation of them by extreme cold. The poisons are preserved also by many antiseptics. Sulphur, creasote, and arsenic, hold these organic poisons in preservation, so that they preserve their active properties.

(n) That some of the poisons are only poisonous during certain stages of their decomposition; with regard to the disease called hospital-fever, there is perhaps only one certain stage when the secretions really contain the poison. There is a certain given stage in the process of the manufacture of the poisons when the secretions change, and at that point the poisonous matter becomes innocuous.

(o) That in considering the development of these poisons it is a common error to suppose that they multiply from a germ as offspring multiply from parents, but that what occurs is this:-Each particle of any one of these poisons brought into contact either with the blood of the living animal or with certain secretions of the living animal, possesses the property of turning the albuminous part of that same blood or that same secretion into substance like itself. The process of change is catalytic. It is a change by which a body is transformed by the presence of some other body which does not itself undergo change. The multiplication of the poison thus takes place through the force of secretion of the person affected, not through the propagation of germ from germ. For instance if poison producing contagious ophthalmia be passed from the eye of one person into the eye of another, presently there is a free secretion. The secretion soon is profuse and is affected by catalysis from the poison. If the inoculation has been deep the whole animal will be affected ; if it has not been deep only the eye will at first be affected. It is not that the particle of poison has propagated a new particle, but it is that the natural secretion of the eyeball has come in contact with a speck of poisonous matter, and immediately at that point where the speck of poison was there is a change in the secretion. This process widens the circle, more secretion pours out and more poison is produced, and the increase goes on until in the end the whole body of the animal may become affected by absorption into the body from the injured surface of poisonous matter: ultimately, i.e., the poison is absorbed into the blood.

(p) That as a general rule the human body furnishes all the poisons that the human body suffers from, that is to say, there is a progression of poison from one body to another, and that ordinary secretions may change and become poisonous without previous infection. This, I showed, had been remarkably brought out in the case of puerperal poison, where a secretion from the hand of the accoucheur had produced puerperal fever. In the case of peritonitis, or inflammation of the peritoneum, there is a secretion which may be carried on the hand of a healthy person and reproduce the disease. Typhus may be produced by the overcrowding of persons in a room through the vaporisation of organic matter at a low temperature. Thus we may have springing up de novo an organic poison which afterwards, on being introduced into one particular body, becomes increased by the secretions of that body.

(q) That as regards the mode in which the organic poisons may be transmitted, they may travel in each of three ways. They may travel by means of sewage as dry solid matter; and all the poisons do this constantly. They may be wafted in the air, or carried by means of linen saturated with the secretions of patients and dried. Again, they may travel in water or in water suspended in the form of vapour.

(r) That the mode of the entrance of organic poison into the body, when they enter by contact, varies with the different poisons, the character of the poison changing the mode of its introduction. The poisons of measles, scarlet fever, and typhus are inhaled. The poisons of small-pox, diphtheria, gianders, erysipelas, hospital-fever, and ophthalmia, require direct contact. The poisons of cholera, yellow fever, and typhoid fever seem always to be swallowed poisons, and may be called, specifically, the poisons of sewage, and therefore mostly travel in a fluid form. They may, nevertheless, travel for short distances as fine dust, and they may travel in water in the form of vapour.

The thought that the poisons of the various spreading diseases are poisonous secretions, and nothing more, came naturally out of my researches. I realised, as it seemed to me, that all these spreading and communicable diseases spring out of the living animal body. That they are as distinctly the offspring of living animals as real progeny are, and that to look to outside sources for them, to look to vegetative growth for them, for example, or seedling, is merely to ignore the basic facts which lie obviously before us for lesson and learning. As well suppose that procreation of animals is due to an external vegetable product or other product dissevered altogether in its origin from the animal as that the poison which creates disease of a communicable kind is in such manner dissevered as to its origin. 
Another thought which occurred to me in the course of my labours, and which I expressed in those earliest records of them, has relation to the force by which the poisons of the various diseases are developed and thrown off. It is well known that the production of the poisons in a living body infected by one of them is limited in respect to duration of time of production even when the body lives and recovers. This fact seemed to me to prove to demonstration that the poison itself is produced by the affected body, and is determined in its production by some natural function of the body or of some part of it. On the basis of my theory, that the poison in every case is a modified secretion, this view of the force of production of the secretion is easily accepted as in accordance with natural law. The force of production is the force of secretion, and so long as the secretion continues changed in character, so long it is thrown off as a poisonous secretion. But so soon as the modification of secretion which rendered it poisonous is stopped, so soon the secretion, flowing onward as before, is rendered innocuous, that is to say, no longer poisonous. If this were not the case, there is no reason, as far as I could see, why, in every instance of infection the infected person should not die. Endow the poison itself with independent forces of life and of reproduction, give to it a distinct reproductive life of its own; then why should it ever cease to reproduce? Why should it not in every case continue to increase within the infected body indefinitely until it kills the body, and why should any one ever recover? But consider the poison as a part only of the animal body itself, a substance to be eliminated from the body by natural methods and then the process of removal of the poisonous condition comes into the natural course of events, and recovery is a natural process, unless some unusual conditions occur to interrupt the natural course.

We see in a common nasal catarrh the outline of this scheme. There is first a dryness of the secreting surface, with reflex nervous irritation and much nervous depression and disturbance thereupon in the circulation of the blood. After a time there is a copious secretion from the nostrils, which continues until the disturbed nervous balance is brought back to steady natural action. At that time the excess of secretion is checked, and nothing more is left than the local effects of hardened secretion or scale due to the desquamation caused by the excessive previous action. In outline this is really the natural course of every epidemic disease, with the exception that the secretion of a catarrh is not definitely proved to be a contagious secretion. I believe it may be so, and sometimes is so; but I need not press the point. The illustration is adduced merely to show that the course of the disease is from within outwards, and that it is checked in its course by restoration of internal natural function. If catarrh were produced by some external vital agency, reproductive in character, lighting upon the nasal tract; if it were due to the colonisation of the nasal tract by an army of foreign invaders which settled there and began and continued to replenish and multiply, when would the catarrh cease? It would, as far as I can see, continue, until by destruction of parts and continued abstraction of secretion and extension of mischief over a wider tract of sur face, it killed inevitably.

A catarrh, according to my view, as it was originally expressed, is typical of all the diseases which run a given course, and are called spreading diseases. It springs up constantly from external atmospheric variations; it runs a given course; it subsides. It is an epidemic, and it would be a true contagious epidemic if the matter secreted from the nasal cavity and the conjunctiva were not so innocuous. As I have hinted already, I believe it may be contagious. I am quite sure that many times in my life I have taken catarrh. by coming near to a person who was affected by it, but whether this contagion is sympathetic or toxic, I am not able to define. On these intimate relations I shall have more to say on a future page.

\section{The Germ Theory.}

In the ten years that have passed since the time named, another hypothesis in reference to the spreading diseases and in relation to their origin from particular poisons has been brought prominently forward. Owing chiefly to the simple name which has been given to this hypothesis, and the commonness of the analogies on which it is based, it has gained much popular favour-I need hardly say that I refer to the so-called germ theory of disease.

This hypothesis has been most prominent for eight or ten years, but it is really a very old speculation indeed, perhaps one of the oldest in medicine. It has its root in the fancy of the analogy that as seed cast on the ground yields, or may yield, a certain harvest after its kind, as a field or garden plot may become fertilised by vegetable seeds or germs which may come to it borne by the atmosphere or by other modes of conveyance, so the body may be infected with germs of disease, which germs, being received in the body as a field for their reception, may increase and multiply in the body, and by their presence excite the phenomena which particularise all the special diseases of a communicable kind.

In modern times Dr. Grove, late of Wandsworth, was the first to advocate this hypothesis, and I need not tell a learned assembly like the present that it has been most energetically advocated more recently by many of the ablest foreign and English men of science. In the course of the discussions and of the researches which have been conducted on this subject much knowledge has, I am sure, been gained in the domain of naiurat history, and much interesting discussion for history has been written on the origin of some forms of life. But I protest that the attempt to connect this knowledge with the phenomena of the various communicable diseases, so as to suggest, or, as some do, to assert, that the diseases in question arise from germs, and that the person affected with a contagious disease has been fertilised like a piece of ploughed land or virgin soil by a crop of germs, and that in turn he is the soil in which another crop is being produced by the independent increasing and multiplying of the germs in him, I protest, I say, that this hypothesis is the wildest, the most innocent, the most distant from the phenomena it attempts to explain, that ever entered the mind of man to conceive. What most astounds me is that men who are conversant with the practice of physic, who are treating diseases of a communicable kind every day, should for a moment connect such a hypothesis. with the phenomena they have under their observation. Does any one of them believe that hydrophobia is from a germ, that syphilis is from a germ, or other diseases I need not specify?

It is suggested by some advocates of the germ speculation that the cause of the communicable diseases is after the manner of the putrefaction of dead organic matter. Does any physician who thinks as he observes, see anything like a general rule of putrefactive change in the contagious diseases? He may of course see local decompositions of secretions and of blood itself in the course of any of the diseases, but these he knows are all secondary results, while he may see and constantly does see all the diseases running their course without any sign whatever of the kind. Nay, in regard to one disease-cholera-he may, as I have done, see it run its fatal course and leave the dead bodies as loth to decompose as if they had been embalmed. Again, does any physician, who thinks as he observes, fail to see that the first symptoms of every one of the contagious diseases are purely nervous symptoms, that they indicate nervous irritation, and that the particular local injuries which occur are not primary at all, 
but are dependent on special nervous 'change modifying nutrition at the part. Between a boil or abscess and a pustule of small-pox what is the difference except in degree of purulent matter formed at one point of formation of matter?

There is nothing whatever in fact in the clinical history of plagues that connects them with the hypothesis of an origin from germs produced without the body and entering it to fertilise it and create a decomposition. When I say there is nothing, I mean there is nothing except the analogy of which I have spoken above, and even that breaks down, for the analogy of the fertilisation of a field by seed means always a definite process of fructification and of results from it; whereas in the history of epidemic plagues there is no such definition.

The germ hypothesis fails, however, on other grounds than the clinical. If it were true that living germs possessing an independent growth and vitality enter the animal body, that every disease of a communicable kind is due to its own external living germ, and that the germs continue to multiply and increase by an independent action of their own; if this be indeed true, why do the germs after a certain time cease to multiply and allow the sick person to recover? Why do they not go on multiplying until the person is infested in every part and fatally stricken? Who would get well from a disease due to living self-propagating contagions? Again, who, if the hypothesis were true, would escape fertilisation? A general fertilising diffusion of self-propagating matter in minute invisible form entering the body as the air may cnter could hardly be expected to select a small minority of a population, and if it did so at the first, why should it clo so when it had seized upon many centres in which it could increase. But the history of all the communicable diseases shows that each epidemic affects individuals individually at different periods in the course of the epidemic according, as a rule, to exposure to the infected, and that the period of the disease is limited by a development and a course rendered in certain periods of time.

I need hardly add in objecting to this germ hypothesis, because the fact is admitted, that not only has no one ever seen a germ of disease, but that no one has ever traced any order of germination in relation to any of the communicable diseases. When a really living self-propagating thing goes through its phases of life and action, like, for example, the yeast growth, we can trace it through its course of action on organic substances, and can study its effects, the changes it produces and the products of such changes. In the epidemic diseases we have no such guidance, no trace of it. Their phenomena, indeed, are opposed to the idea of the self-action of a foreign vital material.

\section{Later Observations on the Glandular Theory.}

I turn again to a brief review of the glandular theory of the origin of the contagious diseases, and of the advances I have made in support of that theory during the period of the past ten years.

In that time $I$ have seen no reason to change my views on the subject of the glandular origin of the communicable diseases. On the contrary, every new observation has tended to confirm it and to make as I think the demonstration of its truth the more definite.

In continuance of observation I have noted that the number of the distinctly communicable diseases is closely related with the number of secretions. The poison of hydrophobia is from the salivary secretion; of diphtheria from the mucous glands of the throat; of scarlet fever I believe from the lymphatic glandular secretion; of glanders from the mucous secretion of the nasal surface; of typhoid from the mucous glands of the intestinal surface; and so on. In some instances the blood itself is infected, and the corpuscular matter becomes the seat of the catalytic change.

A second point which has occurred to me is that the matter or particle which sets up the poisonous action, instead of being living matter, is matter actually dead, and that its effect for evil depends, in fact, upon its being dead. I mean that the dead particles of organic matter in contact with living are the cause of the physical change which transforms the new particles of secretion into poisonous particles as they are brought up to the infected surface to be influenced by the infection.

On the ground that the poisons are always of glandular origin I have been led to the conclusion that under certain influences affecting glandular action the poisons may be made to originate directly through nervous impression without the necessary intervention of an infecting particle. In many epidemics it is common to see a number of examples of the prevailing disease the origin of which is traceable only to fear or anxiety. We call these nervous cases, and we try to define them as such and as distinct from cases due to contagion of a direct kind. But the symptoms are the same as those which follow actual contagion, and in epidemics of cholera they take even a fatal character. My theory explains fully the reason of this. It indicates that an extreme nervous impression acts on the glandular nervous supply, paralyses the glandular function, and thereupon produces the same phenomena as is produced in other instances by the action of a specific poison.

The theory in this manner accounts for the origin of an epidemic disease from an impression made on the nervous system without the direct contact of poisonous matter, as well as for the after-propagation of the disease by distribution of poisonous particles when that is communicated from an infected to a healthy person. It accounts equally well for the production of disease and of a poisonous glandular product under conditions of starvation and cold, by which the nervous tension is reduced. Again, it accounts for the production of disease and of a poisonous glandular secretion under special atmospherical conditions in which the activity of the atmospheric oxygen is reduced in sustaining power.

It has occurred to me further, as a result of the study of the action of the poisonous particles, that when they are brought into contact with the secreting surface, their action towards the body at large is, in the first instance, directly on the nervous fibre. The poisons act in the first instance as irritants on the peripheral nervous surface, and their effect may, I believe, extend particie by particle, as by diffusion, through the whole length of the nervous cord to the nervous centre. I have no doubt this is what slowly takes place in hydrophobia. I believe this is what takes place in diphtheria when paralysis is the sequel of the acute symptoms of the malady. I believe the same mode of progression of the poisonous influence is what happens after inoculation with matter of small-pox, that the severe nervous symptoms which mark the onset of that disease are due to the extensive injury inflicted on the nervous organisation, and that the diffusion of the eruption over so wide a surface of the skin and mucous membrane is the reflex on the peripheral nervous surface from the nervous centres.

It is worthy of special notice in connection with this part of my subject, that in the communicable diseases attended with an eruption on the skin or nervous surface, the eruption, as a rule, takes a circular form. If it be a point of vascular blush, a patechial spot, it is a rounded spot ; if it be a pustule it is rounded ; if it be a more diffused rash it commences in centres which are rounded points. This appearance is an indication of nervous injury. The rounded surface is the radius of injury done to the nervous supply of that part. It is a paralysis of the centre of nervous distribution over the affected part. My researches on the influence of extreme cold on nervous 
unction is strikingly illustrative of this fact. They suggest that the nervous impressions sent from the centres to the peripheral surface when they reach the peripheral surface, as on the surface of the skin, spread out like circling waves, just, in fact, as water spreads out in circles on a pool when a stone is made to impinge on the surface.

Some other recent investigations on the mode of action of the poisons of the communicable diseases has led me to suspect the source of the symptom which is so common to most of them, and which is known as the attendant fever. The fever is of three kinds: primary, reactive, and remittent. The primary fever is that which precedes and attends the eruption of an eruptive disease. The reactive fever is that which succeeds the extreme collapse of an acutely-exhaustive disease like cholera, or an exposure to extreme cold. The remittent fever is that which succeeds upon an acute form of disease, and indicates either that there has been secondary absorption of matter from an abraded surface in contact with poisonous substance, or that some fibrinous or pustular matter has formed within the body, as it were, and become a new and permanent centre of infection.

The first of these forms of fever is, I believe, due to the impression on the nervous centres by the poison in the manner I have described above.

The second, the reactive fever, is, I believe, due to the same action as that which locally may be induced by extreme cold, viz., by an influx of blood into vessels that have been paralysed, and by a rapid radiation of heat from extensive surface of blood.

The third form of fever, the remittent, has an origin, I believe, specifically its own. I have found that pustular matter and all secretions containing fibrinous or cellular structure have the property, by their presence, of liberating oxygen from solution. This extends, as I have found, to blood charged with oxygen, and I am led to the inference that when there is an absorption of such matter into the circulation it causes an undue liberation of oxygen with a quicker combustion, a fever which lasts until the exciting matter is itself destroyed and eliminated, and which does not recur until there is re-absorption of more of the exciting agent. In this physiological mode I should explain all the phenomena of the remittent attack; the cold stage incident to the absorption of the exciting matter; the hot stage incident to the period when, by its presence, the exciting matter is setting free excess of oxygen; the sweating stage when, by rapid elimination through the sweat glands, the equilibrium of temperature is restored.

The study of the glandular theory of the communicable diseases has suggested to me another thought which observation of the diseases fully confirms, viz., that these diseases, like all which have their root in nervous derangement, present a distinct heredity. The impression of disease made on a nervous centre is transmitted. There can be no doubt as to transmission of tendency to particular communicable diseases. Any physician in full practice can find any amount of evidence on the fact by simple natural inquiry. Typhoid fever is clearly a disease possessing hereditary transmissible quality. Diphtheria is the same. Scarlet fever is the same, and small-pox I should suspect was once almost universally so characterised. These facts alone, one of them alone, is sufficient to stamp the origin of the communicable diseases as from the animal body itself. It is certainly one of the best of proofs of the truth of the theory of the glandular origin of the poisons. It will be seen at once by those who look with sufficient patience, that the mode of connection of the diseases in hereditary line is the same as that which connects hereditary qualities of every kind, physical type, mental type, all else that binds many individualities into one family.

Lastly, the study of the glandular theory of the commu- nicable diseases enables me to offer the the most rational explanation of the phenomenon of non-recurrence of the diseases after they have once attacked a person susceptible to them. It is well understood that, as a rule, a person who has been affected by a communicable disease is not affected a second time. To this rule there are many exceptions, but on the whole it holds good. On my theory the reason of the phenomenon is simple enough. They who are susceptible are born with a nervous impression tending to the production of a glan. dular secretion easily changed into poisonous secretion under the direct action of contact with poisonous matter, or even under the influence of a central nervous depression, whereby the glandular function is deranged. But when such a person has passed through the ordeal the tendency, for a time at least, disappears owing to the complete modification of glandular function that has been induced, to the free elimination that has been established, and probably to the change in the nervous matter itself that has resulted from organic modification. Hence the organism becomes susceptible for a time, and if the tendency be not intense that time may mean the whole of the life. Indeed as life advances and nervous susceptibilities derived directly from ancestry lapse into individual self-sustained susceptibilities, these tendencies to disease subside as a general fact, and lose their activity if not their existence.

I turn, in conclusion, to consider for a moment from the view of the glandular theory of the communicable diseases, the practice that is suggested for the suppression of the plagues of mankind.

It will be seen at once that on this point nothing can be wider than the distance between the idea of contagium as a living self-productive thing, reproductive and independent, and the theory of the production of the contagium in each affected person by the force of prociuction of his own secretion. The latest and one of the ablest advocates of contagium vivum, Dr. W. Roberts, says, respecting contagium:-- "We know of nothing that exhibits the phenomena of growth and multiplication except a thing possessed of life."

I admit that readily, but my argument is that the process of secretion is a process of life, and that this living process, perverted as I have described, is amply sufficient to account for the production of all the poisons that exist to cause the communicable diseases, that it accounts for the number of these diseases, and more, that it accounts for the limitation of the number.

\section{The Glandular Theory in its Application to Practical Sanitation.}

The practical usefulness of the theory, however, con. sists in its direct application. If the contagium vivum view be true, if the air around us is charged with invisible germs which come from whence we know not, which have unlimited power to fertilise, which need never cease to fertilise and multiply, what hope is there for the skill of man to overcome these hidden foes? Why on some occasion may not a plague spread over the whole world, and destroy its life universally?

My theory presents an altogether different aspect. I say to living men and women, it is you who are the producers of the communicable diseases, or if it be not you yourselves it is one of your lower earthmates in creation, some domestic animal that shares with you the power of producing a poisonous secretion and of giving a hereditary stamp of production to such poisonous product. I look on the man or animal affected with a contagious disease as one precisely, for the time, in the position of the cobra or other animal that naturally secretes a poison, and recognising this fact I see at once that the danger is all but limited to the person affected.

Isolate that person from the rest of mankind, take care that his secretions, volatile, fluid, or solid, do not come into 
contact with the secretions of susceptible healthy persons, and the danger is over. With the recovery of that person, that is to say, with restoration in him of a natural secretive process, the poison is destroyed; or should he unfortunately die, then with the death of his power to produce further secretion the danger is over, unless from his dead body some of the poison formed before the death be actually carried away to infect. In a word, if my theory be true, we sanitarians have complete mastery over the diffusion of the poisons of all the communicable diseases. We have but to keep steadily in view that the producing and reproducing power is in the affected body, and we can, even with our present knowledge, all but completely limit the action to the propagating power of that body-its power, I mean, of secretion and diffusion of secretion.

Beyond this, if the theory be true, we must expect, as we reduce the communicable diseases of one generation to reduce the tendency to them in the next generation, so that in time the heredity to particular spreading disease shall be thoroughly wiped out.

The theory suggests a profitable line of research on the subject of the production and reproduction of some of the poisons by the inferior animals and their transmission in that course to man. It brings all the inferior animals, in respect to their health and comfort, under our especial human care, not only for their sakes, but for our own self-preservation.

Finally, the theory suggests to those who are engaged in treating diseases of a communicable kind the best means of arresting the progress of a communicable disease even when the phenomena of it have been developed in an individual. It leads us physicians to take a precise view, in each such case, of the nervous and glandular processes that are out of the natural order of work; it suggests to us to seek for remedies amongst chemical agents which affect special secretions; and it shows us how to place the sick under silch conditions that the secondary absorption of their own poisonous secretions, - that deep absorption which, according to my experience, is the actual cause of death in the great majority of cases of contagious disease,-may be avoided.

In every direction, in fine, in prevention and in cure, the glandular theory of the origin of the communicable diseases opens practical work and hopeful work.

I have for some time past sought for a favourable opportunity of once more putting forward this theory of the natural origin and cause of the communicable diseases of men and animals. The present is opportune to the fullest degree, and therefore I have seized on it. I am too earnest after search of truth for its own sake, too certain that in science everything false must fall, and everything true must remain, to feel any sense of anxiety as to the fate of my simple theory, by the side of the doctrine of a living contagium. If my doctrine be as true as I believe it to be, it will live, whatever force be arrayed against it. If it be not true, I would be of the first to welcome its end, and to hail the ascendency of what is absolutely provable and certain on the momentous questions that have occupied our attention.

Meantime, I know I could not do a better thing for my own views than submit them once more to the public eye through the audience which has now so attentively listencd to the argument.

\section{NOTES}

THERE has been a great deal of talk during the last few rlays, by prominent public men, on the advantages of some equivalent for university education for all the people, an education, too, in which science would be allotted a just place. Last week a Nottingham, the Earl of Carnaryon and Mr.' Gladstone said much that was at least true on the advantages of an institution such as that newly founded at Nottingham, and each from his own standpoint lauded the advantages of wide culture for all classes.
Both Mr. Gladstone and,Mr. Forster on Tuesday at Bradford seemed distinctly to approve of the movement for creating Owens College a University; and the only difficulty now seems to be the question of power to grant degrees. But surely those who are so eager on the latter point forget to distinguish between the shadow and the substance; the question of degrees will no doubt settle itself after the University has been established. Still we hardly sympathise with the trade-mark view of degrees propounded by Dr. Appleton in the Times. Bass's or Allsopp's label is imitated because their ales have a high and no doubt well-deserved reputa. tion. But there were good ales before the names of either of the Burton brewers were heard of ; there is the fine old Oxford ale, for instance, which, to judge by the public taste, has been improved upon by its new Burton rivals. Mr. Forster, however, we must say, seemed to think Oxford deserving of a word of praise for its present activity. Mr. Forster's address at Bradford was no mere essay on the beanty of culture, but the weighty utterance of a "practical" man who is forced to confess that he daily feels the immense disadvantage of having had no early training in science. He produced himself, in fact, as a practical comment on Sir John Lubbock's previous advocacy of the introduction of science into elementary schools. "His ignorance of science," he said, "his want' of having been taught elementary laws of science when a boy, he felt every hour of his life, and it was too late now to learn. Science, if leant at all, must be learnt in boy hood, and it was really disgraceful that in this civilised country, in this intellectual age, any one should be brought up in ignorance of the laws of nature, upon the breaking or keeping of which depended our happiness, our lives, and almost everything that relates to us. What a loss of pleasure, and what a different world the outside world of nature would be to him, if he could look around and understand the meaning of the various forces which were at work; and there was no dutubt that a boy, even at an elementary school, if he learnt the elements there and went on afterwards, would get that kind of knowledge of the laws of science that it would become easy to him. There was a great talk about the dead languages. He was not going to say anything against them. Latin was almost a necessity to a man of culture, and Greek was of use ; but why should nature, whichspoke to us in so many ways, be a dead langtiage to us? And therefore, if it came to this question-Whether we were to have classes on special subjects in elementary schools, classes for grammar, predicat $\mathrm{C}$, and a great many long words which he hoped nobody would examine him in, or for science-he certainly should go in favour of science." These are weighty words coming from a man of Mr. Forster's experience and "common sense," and indeed make us hope that things are progressing, and that we shall not now have long to wait before science is introduced not only into colleges, but into schools of all grades. Mr. Forster concluded by admitting that the German workers were superior to ours in the fact that they added to practical training scientific knowledge, and that he saw no reason why in secondary and even university education voluntary efforts should not be seconded by State aid.

M. Yvon VILlarceau has been appointed "Administrateur Provisoire" of the Paris Observatory by an order of the Minister for Public Instruction, dated Saturday last. M. Villarceau held a similar office after the death of Delaunay, before the reappointment of Leverrier. Nothing has been said yet as to the appointment of a successor.

A $\mathrm{T}$ the Guy's Hospital conversazione, on Monday evening, a new government filter, invented by Major Crease, was shown, which reduced strong tea and infusions of logwood to clear tasteless water. The nature of the filtering material is not made known.

THE white whale, which was brought from America and placed in a tank (50 feet by 25 ) of fresh water in Westminster 